\title{
Research on Harmonic Problem of Micro-grid with Distributed Grid Connected Inverter
}

\author{
Xiangzheng Zhao, Dongmei Zhao \\ North China Electric Power University, Beijing 102206, China \\ 18631997789@163.com
}

Keywords: Distributed generation, Harmonic resonance, Power quality.

\begin{abstract}
With the grid connected inverter is widely used in the new energy power generation, which is represented by wind energy and photovoltaic power generation, the resonance problem caused by the grid connected inverter becomes more and more a threat to the safe and stable operation of power system. This paper firstly introduces the basic structure of the new energy grid connected system, which is represented by wind power and photovoltaic system. Secondly, the harmonic resonance of the new energy grid connected system is analyzed. Finally, this paper introduces the optimization operation control process of the multi-function grid connected inverter, and proposes a power quality and reliability management strategy based on summarizing the advantages and disadvantages of various methods.
\end{abstract}

\section{Introduction}

Over the past decade or so, the deteriorating environment has been associated with the non-renewable nature of traditional fossil fuels and the public's concerns about the safety of nuclear power generation, which has contributed to the sustained and rapid development of renewable energy sources such as wind and solar energy. However, these new energy power generation forms are intermittent and random operation characteristics, unstable output characteristics and uncontrollable source power to the safe and stable operation of the power system has a negative impact, so the urgent need for large-scale new Energy after the impact of the system to study[1-3]. On the basis of new energy power generation, the concept of distributed power generation system is further developed. Traditional power systems use high-power, centralized power generation, but the new energy power generation has the characteristics of decentralized resources, so it is more suitable for decentralized access to the grid. Compared with the off-grid work, grid-style scenery system without additional energy storage device, not only can save power and operating costs, but also can improve grid efficiency. However, the distributed power supply issued by the DC can't be directly supplied to the grid and AC load, must go through a certain interface device that the inverter can be achieved in parallel [4,5]. Grid-connected inverter system has a flexible and efficient features, but grid-connected inverter also brings new challenges to the grid.

\section{Grid - Connected Mechanism of Distributed Inverter System}

Grid-connected inverter is the core component of the grid-connected system. According to the type of DC-side power supply, can be divided into voltage source and current source inverter. In the distributed power generation system, the working mode of the grid-connected inverter can be divided into grid-connected mode and island mode [6].

Inverter output filter is the role of power electronic switching devices to produce high-order harmonic filter, in addition to isolation and energy storage auxiliary function. At present, the grid-type inverter filter types are L-type, LC type, LCL type, LLCL type [7]. 


\section{Inverter Harmonic Analysis and Optimized Compensation Control}

Figure 3 shows a typical feeder branch of the microgrid, which consists of a three-phase, two-level, multi-function grid-connected inverter, a local non-linear and reactive load, and the other parts of the microgrid are equivalent to a set of voltages source. Among them, the grid inverter by the equivalent of the DC voltage source, DC bus capacitor, bridge circuit, filter inductance composition [8].

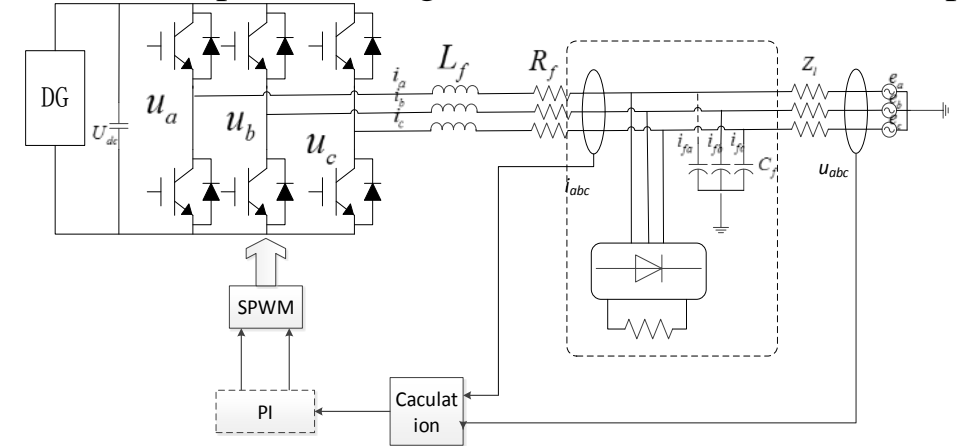

Fig1. Multi-function grid connected inverter in micro grid

As the three-phase two-level grid-connected inverter compensation for unbalanced load current capacity is not strong, here mainly consider the harmonic and reactive current compensation. The model is based on the two indexes of harmonics and power factor of grid-connected current at PCC. Assuming that the harmonics have a slightly stronger effect on the micro-grid than the reactive power, the comparison matrix is chosen:

$$
C=\left[\begin{array}{ll}
1 & 3 \\
\frac{1}{3} & 1
\end{array}\right]
$$

Find the eigenvalues and eigenvectors of the matrix, and take the consistency index:

$$
C I=\frac{\lambda_{\max }-n}{n-1}
$$

And $\lambda_{\max }=2$ is the largest eigenvalue of matrix $C$ and $n=2$ is the order of $C$. If $C l=0$, consistency test passed. The weight vector corresponding to the maximum eigenvalue $\lambda$ max is normalized to obtain the weight vector $\omega=\left(\omega_{1}, \omega_{2}\right)=(0.75,0.25)$.

In the optimization control of multi-function grid-connected inverter, because of the limited capacity of the input, it can only be more reference to the optimization target, there is a certain proportion of reactive power and harmonic compensation current. The optimal compensation control strategy for the multifunctional grid-connected inverter is determined in two different ways to determine the compensation coefficients $a_{1} a_{2}$ in the optimal compensation control strategy.

Multi-function grid-connected inverter compensation capacity can be expressed as:

$$
S=\sqrt{S_{h}^{2}+S_{q}^{2}}=3 U_{p} \sqrt{\left(a_{1} I_{h 0}\right)^{2}+\left(a_{2} I_{q 0}\right)^{2}}
$$

Among them, Up is the phase voltage RMS, $S_{h} S_{q}$ are the multi-function grid inverter input harmonic and reactive power compensation capacity, $\mathrm{I}_{\mathrm{h} 0}, \mathrm{I}_{\mathrm{q} 0}$ are compensated before the multi-function grid inverter and the network out of the harmonic Wave, reactive current effective value, $\mathrm{a}_{1} \mathrm{a}_{2}$ are multi-function grid inverter input harmonics, reactive current accounted for the detected harmonic and reactive current proportional coefficient. We can select the objective function formula (4) of the optimal compensation model, which is subject to the conditional constraint of (5):

$$
\begin{gathered}
\min F_{1}=a_{1}^{2} I_{h 0}^{2}+a_{2}^{2} I_{q 0}^{2} \\
\left\{\begin{array}{l}
I_{h 0}=\psi_{h 0} I_{1} \\
I_{q 0}=\psi_{q 0} I_{1}
\end{array}\right.
\end{gathered}
$$

Then $\psi_{h 0}$ is the total harmonic distortion rate of the compensated front-net current and $\psi_{g 0}$ is the reactive coefficient of the current before the grid is compensated. The reactive power factor is defined 
as the ratio of reactive power to quarterly apparent power, that is, the ratio of reactive current to fundamental current $\mathrm{I}_{1}$ :

$$
\psi_{q}=\frac{Q}{3 U_{p} I_{1}}=\frac{3 U_{p} I_{q}}{3 U_{p} I_{1}}=\frac{I_{q}}{I_{1}}
$$

Assuming that the THD and the reactive power of the grid current after compensation are $\psi_{h} \psi_{g}$, then the power quality index at this time is:

$$
\omega_{1} \psi_{h}+\omega_{2} \psi_{q}=A
$$

And $\mathrm{A}$ is the set target constant. On the one hand, the power quality at the microcomputer PCC should meet the corresponding standards for the grid, such as THD $<5 \%$ and PF $>0.98$. Therefore, in this case, the power quality control object A can be set directly according to the power quality standard. On the other hand, grid-connected inverter to the power grid to provide more power quality management and reactive support and other ancillary services. In this case, the power quality control target A may be set according to the dispatch instruction of the grid. In general, the fundamental current component changes little before and after compensation, in the analysis to facilitate consideration, that I1 before and after the compensation is similar. Ideally, the relationship between THD and reactive power before and after compensation is satisfied:

$$
\begin{aligned}
& \left\{\begin{array}{l}
\psi_{h}=\left(1-a_{1}\right) \psi_{h 0} \\
\psi_{q}=\left(1-a_{2}\right) \psi_{q 0}
\end{array}\right. \\
& L_{1}=a_{1}^{2} I_{h 0}^{2}+a_{2}^{2} I_{q 0}^{2}+\lambda_{1}\left(\omega_{1} \psi_{h}+\omega_{2} \psi_{q}-A\right)
\end{aligned}
$$

And $\lambda_{l}$ is the Lagrangian multiplier. Note that if there is no power quality compensation, $L_{l}=0$ $a_{l}=a_{2}=0, A=\sigma$. On the contrary, when the harmonics and reactive power in the micro-grid are fully compensated, then $L_{l}=I^{2} h 0+I_{q 0}^{2} a_{l}=a_{2}=1, A=0$. Thus, the Lagrangian multiplier has a range $\left[0, I^{2}{ }_{h 0+}\right.$ $\left.I_{q 0}^{2}\right]$. The partial derivative of each variable can be obtained:

$$
\left\{\begin{array}{c}
\frac{\partial L_{1}}{\partial a_{1}}=2 a_{1}\left(\psi_{h 0} I_{1}\right)^{2}-\lambda_{1} \psi_{h 0} \omega_{1}=0 \\
\frac{\partial L_{1}}{\partial a_{2}}=2 a_{2}\left(\psi_{q 0} I_{1}\right)^{2}-\lambda_{1} \psi_{q 0} \omega_{2}=0 \\
\frac{\partial L_{1}}{\partial a_{2}}=\omega_{1} \psi_{h}+\omega_{2} \psi_{q}-A=0
\end{array}\right.
$$

Then it can be solved:

$$
\left\{\begin{array}{c}
a_{1}=\sigma-A \omega_{1} /\left[\left(\omega_{1}^{2}+\omega_{2}^{2}\right) \psi_{h 0}\right] \\
a_{2}=\sigma-A \omega_{2} /\left[\left(\omega_{1}^{2}+\omega_{2}^{2}\right) \psi_{q 0}\right] \\
\lambda_{1}=2(\sigma-A) I_{1}^{2} /\left(\omega_{1}^{2}+\omega_{2}^{2}\right)
\end{array}\right.
$$

Among them, $\sigma$ is not compensated when the grid at the power quality increase and evaluation indicators, namely:

$$
\sigma=\omega_{1} \psi_{h 0}+\omega_{2} \psi_{q 0}
$$

According to the above analysis results, the optimal compensation coefficient can be obtained. When the power quality index reaches the full compensation (corresponding to the compensation coefficient is 1), it is considered that the power source has no such problem. The weight of the mass impact factor is calculated as 1 , and the optimal compensation coefficient is recalculated.

In this paper, a comprehensive evaluation method of microgrid power quality based on AHP is used and applied to the optimal compensation control of multi-function grid-connected inverter. Based on the comprehensive evaluation index of power quality, the optimal compensation control strategy of multi-function grid-connected inverter is put forward, and the power quality is optimized by inputting the given compensation capacity respectively to achieve the minimum compensation capacity for a given power quality index aims. 


\section{Summary}

With the continuous development of distributed inverter system in China, the grid operation has put forward new challenges to the development of existing distribution network, which increases the difficulty of planning, designing and controlling the distribution network. Therefore, through the control of distributed inverter system and harmonic suppression, which can effectively reduce the impact of a large number of new energy access to the power distribution network, it is helpful to improve the power quality and maintain the safe operation of the distribution network. Considering the increasing proportion of distributed new energy power generation in the distribution network, the distributed power generation system has the ability of providing the auxiliary service to the power grid, such as reactive power / voltage dynamic support ability. In the active distribution network, large-scale distributed inverter system should be passive to active, with more active control function, according to the grid operating state adaptive adjustment mode of operation. Therefore, we can further study the active distribution network with multi-functional, adaptive distributed inverter system operation and control strategy.

\section{References}

[1] Zhao Zhengming, Lei, he Fanbo, etc. Large capacity grid connected photovoltaic power plant technology: a review [J]. Automation of Electric Power Systems, 2011, 35 (12):101-107.

[2] International Energy Agency. Technology Roadmaps China wind energy development roadmap 2050. Paris: IEA, 2011

[3] Zaininger H W,Bell D J.Potential dynamic impacts of wind tubines on utility systems[J].IEEE Trans on Power Apparatus and Systems, 1981,100(12):4821-4829.

[4] Papadopoulos.Simulation and analysis of small and medium sia power systems containing wind turbines[J].IEEE Trans on Power Apparatus and Systems,1991,6(4):1453-1458.

[5] Shen Hong, Liang Jun, Dai Huizhu. Power system transient stability analysis based on wind farm penetration power limit calculation [J]. Power system technology, 2002, 26 (8):8-11.

[6] Wiik J,Jgjerde O,Gjengedal T,et al.Steady state power system issues when planning large wind farms[C].IEEE Power Engineering Society Winter Meeting,New York,2002.

[7] Zheng Guoqiang, Bao Hai, Chen Shuyong. An improved algorithm for wind farm penetration power limit optimization based on approximate linear programming [J]. Proceedings of the CSEE, 2004, 24 (10):68-71.

[8] Tao Shun, Xiao Xiangning. Based On A Short Board Effect Level Of Power Quality Comprehensive Evaluation [J]. Electric Power Technology, 2008, (02) : 16-20. 